\title{
Electromagnetic mass difference on the lattice
}

Yusuke Namekawa*

Department of Physics, Nagoya University,

Nagoya 464-8602, Japan

E-mail: namekawaleken.phys.nagoya-u.ac.jp

\section{Yoshio Kikukawa}

Department of Physics, Nagoya University,

Nagoya 464-8602, Japan

E-mail: kikukawa@eken.phys.nagoya-u.ac.jp

We calculate electromagnetic mass difference of mesons using a method proposed by Duncan et al. The RG-improved gauge action and the non-compact Abelian gauge action are employed to generate configurations. Quark propagators in the range of $m_{P S} / m_{V}=0.76-0.51$ are obtained with the meanfield-improved clover quark action. Chiral and continuum extrapolations are performed and the results are compared with experiments. Finite size effects are also examined. Quark masses are extracted from the measured spectrum. Our preliminary values for light quark masses are $m_{u}^{\overline{M S}}(\mu=2 \mathrm{GeV})=3.03(19) \mathrm{MeV}, m_{d}^{\overline{M S}}(\mu=2 \mathrm{GeV})=4.44(28) \mathrm{MeV}$, $m_{s}^{\overline{M S}}(\mu=2 \mathrm{GeV})=99.2(52) \mathrm{MeV}$.

XXIIIrd International Symposium on Lattice Field Theory

25-30 July 2005

Trinity College, Dublin, Ireland

${ }^{*}$ Speaker. 


\section{Introduction}

Numerical simulations based on lattice QCD allow us to calculate physical quantities in high accuracy [1]. However, electromagnetic and isospin-violating effects are usually ignored. Taking into account these effects is important for more realistic predictions. In practice, it is pointed out that for light quark masses systematic errors from electromagnetic effects can be comparable with statistical one [2].

An attempt was made to include electromagnetic effects in lattice QCD [3]. Pseudoscalar meson masses are calculated on the background of gluon and photon fi elds. $\pi^{+}-\pi^{0}$ splitting is found to be 4.9(3) MeV at $\beta=5.7$ with the Wilson action. Their result shows a good agreement with experiments. But their simulations were performed at a single lattice spacing with unimproved actions. The scaling violation of their result is not expected to be small. Another point is that the fi nite size corrections were estimated only with a model. It is desirable to evaluate fi nite size effects from a fi rst principle calculation directly.

In this work, we study electromagnetic mass splittings of mesons and extract quark masses in the quenched theory. 1 We employ an improved action combination, the renormalization group $(\mathrm{RG})$ improved gauge action and the meanfi eld-improved clover quark action. Pseudoscalar and vector meson masses are computed at three lattice spacings and extrapolated to the continuum limit. Finite size effects are evaluated on lattices with the spacial size of $L=2.4 \mathrm{fm}$ and $L=3.2 \mathrm{fm}$. Comparison of $m_{\pi}^{+}-m_{\pi}^{0}$ as well as $m_{\rho}^{+}-m_{\rho}^{0}$ with experimental values are made. Electromagnetic contribution to kaon mass splittings is also discussed.

\section{Method}

We generate $S U(3)$ and $U(1)$ fi elds and calculate quark propagators on the combined $S U(3) \times$ $U(1)$ confi gurations. $S U(3)$ confi gurations are generated by the pseudoheat bath algorithm. For the $U(1)$ gauge part we employ a non-compact Abelian gauge action. $U(1)$ confi gurations are constructed of the Fourier transformed photon fi elds in the momentum space [5]. We fi $x$ the gauge to the Coulomb one in both $S U(3)$ and $U(1)$ parts and use a gaussian smearing function. Meson masses are computed from quark propagators. In this calculation, we use only connected part of quark propagators. In the presence of electromagnetic fi elds, isospin is no longer a conserved quantity. Though the violation for $m_{\pi^{0}}^{2}$ are expected to be small, disconnected diagrams may give measurable contribution. Estimate of the disconnected part is under investigation. Masses are obtained by $\chi^{2}$ fi ts to hadron correlators, taking account of correlations among different time slices. Statistical errors of hadron masses are estimated with the jack-knife procedure.

Our simulations are performed at $\beta=2.187\left(a_{\rho}=0.20 \mathrm{fm}\right), \beta=2.334\left(a_{\rho}=0.16 \mathrm{fm}\right)$ and $\beta=2.575\left(a_{\rho}=0.11 \mathrm{fm}\right)$ using $12^{3} \times 24,16^{3} \times 32$ and $24^{3} \times 48$ lattices with the spatial extent $L \sim$ $2.5 \mathrm{fm} .16^{3} \times 24$ lattices at $\beta=2.187$ are also employed to examine the fi nite size effects. We take four hopping parameters corresponding to $m_{P S} / m_{V}=0.76-0.51$. Confi gurations are generated independently at each $m_{P S} / m_{V}$. Measurements are carried out at each 100 sweeps. Our simulation parameters are summarized in Table 1. These parameters are chosen so that they correspond to those of two-flavor full QCD data generated by CP-PACS.

\footnotetext{
${ }^{1}$ For a similar work using $N_{f}=2$ DBW2 gauge and domain wall fermion actions, we refer to Ref. [4].
} 
Table 1: Simulation parameters

\begin{tabular}{l|ccc}
\hline$\beta$ & 2.187 & 2.334 & 2.575 \\
\hline Size & $12^{3} \times 24\left(16^{3} \times 24\right)$ & $16^{3} \times 32$ & $24^{3} \times 48$ \\
\hline$N_{\text {conf }}$ & $800(400)$ & 400 & 100 \\
\hline
\end{tabular}
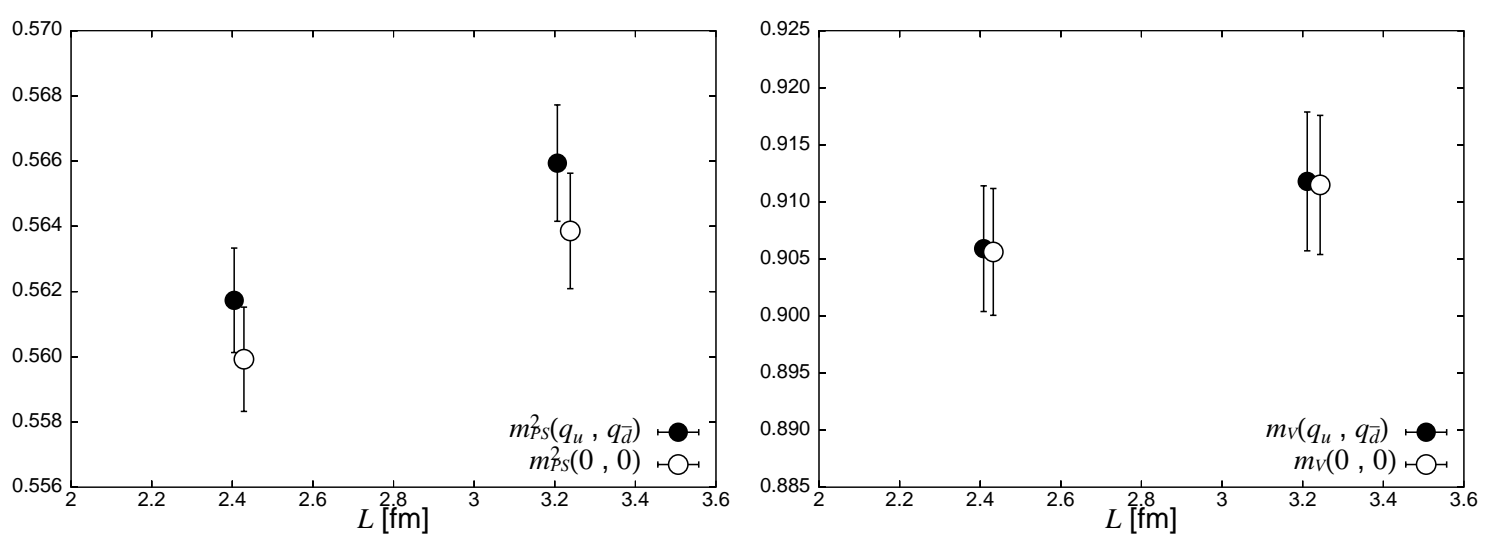

Figure 1: Volume dependence of charged pseudoscalar (left panel) and vector meson masses (right panel) at $m_{P S} / m_{V}=0.62$ on $12^{3} \times 24$ lattice. For comparison, masses in pure QCD (open symbols) are also plotted.

\section{Simulation results}

We fi rst check fi nite size effects in our results. Finite size corrections may be enhanced in the presence of electromagnetic fi elds because electromagnetic fi elds have a long interaction range. It is necessary to estimate magnitude of fi nite size corrections. In Fig. 1, we plot charged pseudoscalar and vector meson masses as a function of the spatial volume. The results obtained on $12^{3} \times 24$ and $16^{3} \times 24$ lattices are mutually consistent within errors. We did not fi nd any enhancement of fi nite size effects by electromagnetic fi elds. In the quenched approximation, $L=2.4 \mathrm{fm}$ seems to be enough for meson calculations, even if there is an electromagnetic interaction.

In order to extrapolate our results to the chiral limit, we fit a hadron mass as a function of quark masses and charges. We employ the following form for a chiral extrapolation of pseudoscalar meson masses.

$$
\begin{aligned}
m_{P S}^{2}= & A_{P S}\left(q_{q}+q_{\bar{q}}\right)^{2}+B_{P S}\left(q_{q}, q_{\bar{q}}\right)\left(m_{q}+m_{\bar{q}}\right) \\
& B_{P S}\left(q_{q}, q_{\bar{q}}\right) \equiv B_{0}^{P S}+B_{2}^{P S}\left(q_{q}+q_{\bar{q}}\right)^{2}
\end{aligned}
$$

where $A_{P S}, B_{0}^{P S}, B_{2}^{P S}$ and $\kappa^{c}$ in $m_{q}, m_{\bar{q}}$ are fi tting parameters. $q_{q}$ is an electric charge for a particle in units of $e$ and $q_{\bar{q}}$ is for an antiparticle. $q=+2 / 3$ for the up quark and $q=-1 / 3$ for the down quark are assigned. We use a quark mass defi ned through a vector Ward identity,

$$
m_{q}=\frac{1}{2}\left(\frac{1}{\kappa_{q}}-\frac{1}{\kappa_{q}^{c}}\right), m_{\bar{q}}=\frac{1}{2}\left(\frac{1}{\kappa_{\bar{q}}}-\frac{1}{\kappa_{\bar{q}}^{c}}\right),
$$




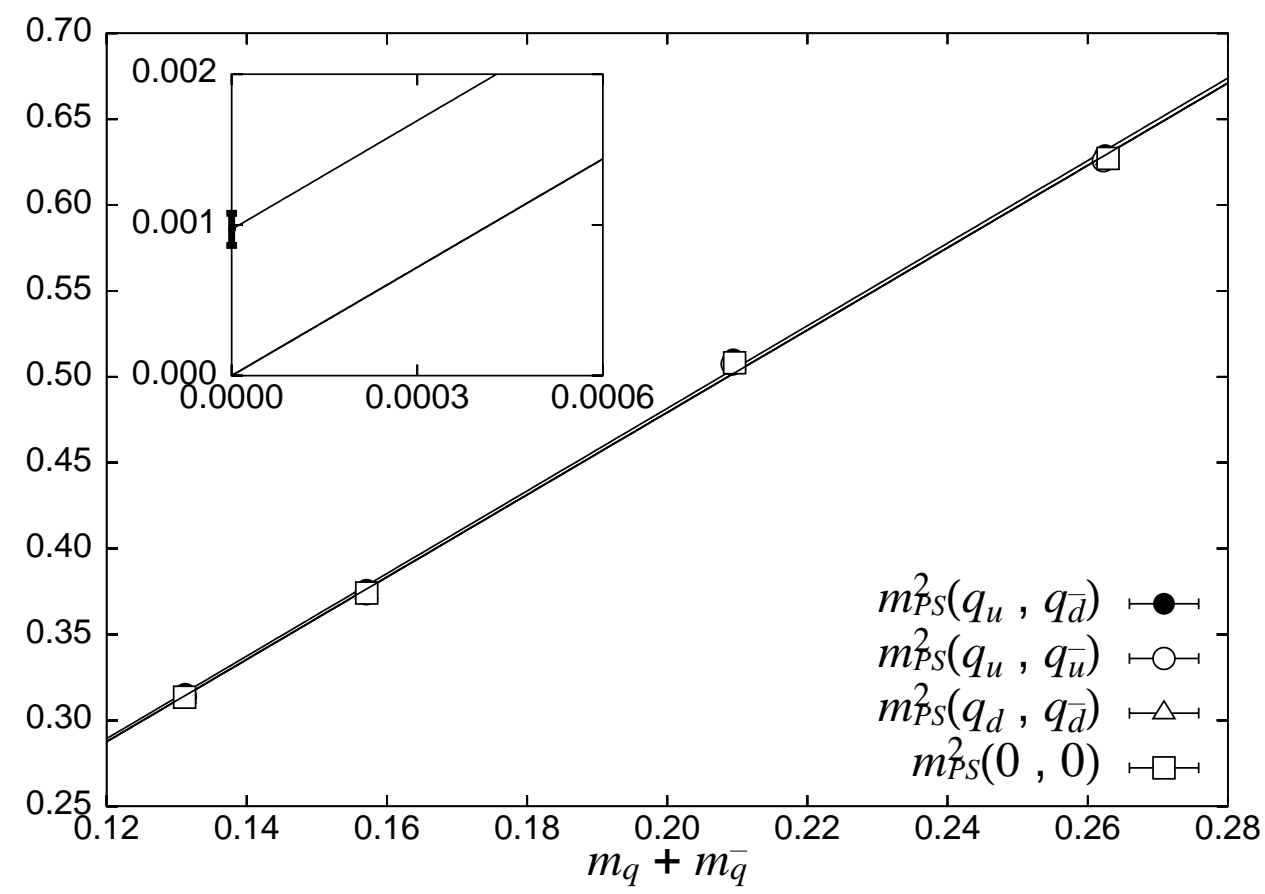

Figure 2: Chiral extrapolations of pseudoscalar meson masses at $\beta=2.187$.

where $\kappa_{q}$ is a hopping parameter for a quark and $\kappa_{\bar{q}}$ is for an antiquark. $\kappa_{q, \bar{q}}^{c}$ are the corresponding critical hopping parameters. This extrapolation function, inspired by the chiral perturbation theory, only depends on the total charge and the quark mass combination. In the fi ts, correlations among several charge combinations are neglected for simplicity. We estimate the errors by the jackknife method. Strictly speaking, the quenched chiral logarithm term must be added to Eq. (3.1). However, the logarithmic curvature is not seen in our data within $m_{P S} / m_{V}=0.76-0.51$. Smaller quark mass data are needed for a more precise extrapolation. We found Eq. (3.1) reproduce our lattice data well. As an example, the fi t result at $\beta=2.187$ is presented in Fig. 2. $\pi^{+}$is heavier than $\pi^{0}$, as we see in nature.

Vector meson masses are extrapolated as follows.

$$
\begin{aligned}
m_{V}= & A_{V}\left(q_{q}, q_{\bar{q}}\right)+B_{V}\left(q_{\bar{q}}, q_{d}\right)\left(m_{q}+m_{\bar{q}}\right), \\
& A_{V}\left(q_{q}, q_{\bar{q}}\right) \equiv A_{0}^{V}+A_{2}^{V}\left(q_{q}+q_{\bar{q}}\right)^{2}, \\
& B_{V}\left(q_{q}, q_{\bar{q}}\right) \equiv B_{0}^{V}+B_{2}^{V}\left(q_{q}+q_{\bar{q}}\right)^{2} .
\end{aligned}
$$

$A_{0}^{V}, A_{2}^{V}, B_{0}^{V}$ and $B_{2}^{V}$ are fi tting parameters. In contrast to the case of pseudoscalar mesons, mass difference of charged and neutral vector mesons is found to be small. This tiny mass difference is consistent with the result calculated by the hidden local symmetry formulation, $\Delta m_{\rho}=-1 \mathrm{MeV}$ [6].

We identify the physical point with experimental values of $\pi^{0}$ and $\rho^{0}$ masses, $M_{\pi^{0}}=0.1350 \mathrm{GeV}$ and $M_{\rho^{0}}=0.7751 \mathrm{GeV}$. By solving Eq. (3.1) and Eq. (3.4) using $M_{\pi^{0}}, M_{\rho^{0}}$, a sum of bare up and down quark masses at the physical point, $\left(m_{u}+m_{d}\right)_{\text {phys }}$, are determined. The lattice spacing $a_{\rho}$ is set by identifying $M_{\rho^{0}}$ with $m_{V}$. Substituting $\left(m_{u}+m_{d}\right)_{\text {phys }}$ to Eq. (3.1) predicts $\pi^{+}$mass. Similarly, Eq. (3.4) gives $m_{\rho^{+}}$. To determine up, down and strange quark masses independently, we 

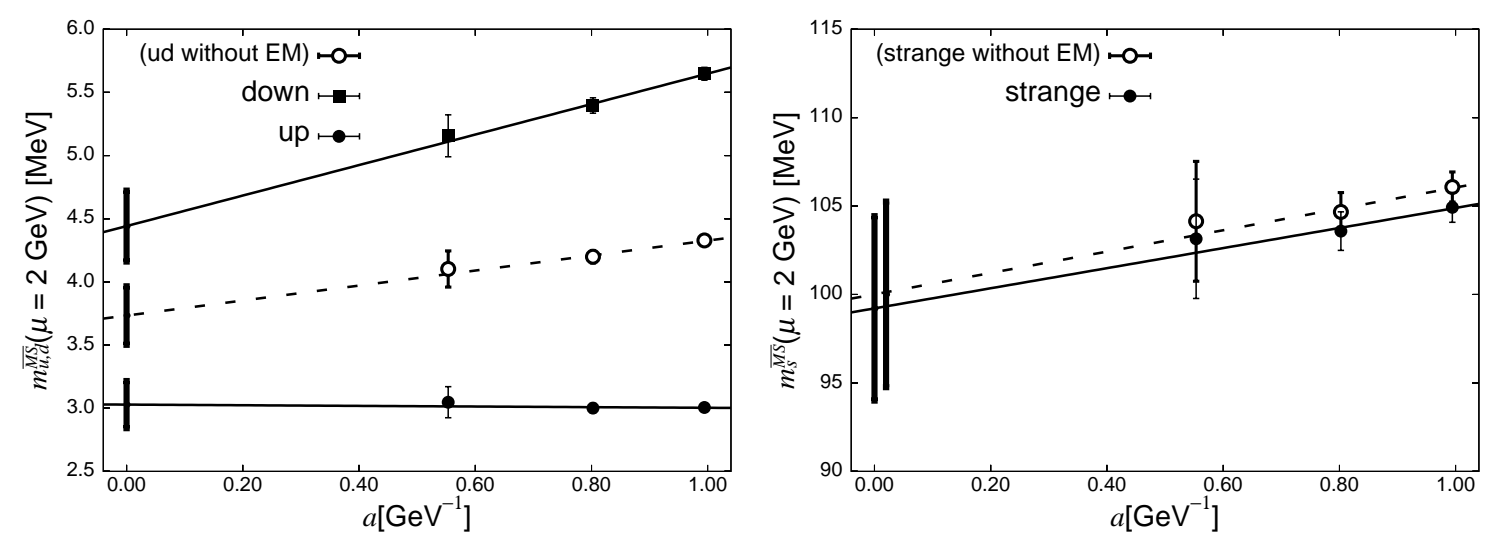

Figure 3: Lattice spacing dependence of quark masses with and without electromagnetic effects.

also use experimental values of $K_{+}$and $K_{0}$ meson masses as inputs. $\left(m_{u}+m_{s}\right)_{p h y s}$ is obtained from $M_{K_{+}}=0.4937 \mathrm{GeV}$ and $\left(m_{d}+m_{s}\right)_{\text {phys }}$ from $M_{K_{0}}=0.4976 \mathrm{GeV}$. Quark masses are renormalized using one-loop renormalization constants $Z_{m}$ and coeffi cients $b_{m}$ at $\mu=1 / a$ [7],

$$
m_{q}^{\overline{\mathrm{MS}}}(\mu=1 / a)=Z_{m}\left(1+b_{m} \frac{m_{q}}{u_{0}}\right) \frac{m_{q}}{u_{0}}
$$

where $u_{0}$ is a tadpole factor, $u_{0}=(1-0.8412 / \beta)^{1 / 4}$. The $\overline{\mathrm{MS}}$ quark masses at $\mu=1 / a$ are evolved to $\mu=2 \mathrm{GeV}$ using the four-loop beta function.

Continuum extrapolations are performed by linear fi ts to the data at three lattice spacings. Our preliminary results for light quark masses are $m_{u}^{\overline{M S}}(\mu=2 \mathrm{GeV})=3.03(19) \mathrm{MeV}, m_{d}^{\overline{M S}}(\mu=$ $2 \mathrm{GeV})=4.44(28) \mathrm{MeV}, m_{s}^{\overline{M S}}(\mu=2 \mathrm{GeV})=99.2(52) \mathrm{MeV}$. Fig. 3 illustrates lattice spacing dependence of quark masses with and without electromagnetic effects. We found electromagnetic contributions to the strange quark mass is $1 \%$. In contrast to the case of quark masses, electromagnetic mass splittings of pseudoscalar and vector mesons show mild lattice spacing dependence. Therefore, we employ constant fi ts for continuum extrapolations of electromagnetic mass splittings. Our results are represented in Fig. 4. The obtained mass splittings are consistent with experimental values. In addition to $\pi^{+}-\pi^{0}$ and $\rho^{+}-\rho^{0}$ mass difference, we can estimate electromagnetic contribution to $K^{+}-K^{0}$ mass difference by Eq. (3.1). Using a constant continuum extrapolation again, the electromagnetic contribution to $K^{+}-K^{0}$ mass difference is evaluated to be $1.420(24) \mathrm{MeV}$, which is close to the value of Dashen's theorem $1.3 \mathrm{MeV}$ [8], rather than a model estimate $2.6 \mathrm{MeV}$ [9]. But, our simulations are in the quenched approximation and the chiral logarithm is neglected. Including dynamical quarks and chiral logarithm effects is needed for a more precise comparison with other calculations.

\section{Conclusions}

We calculated electromagnetic mass splittings using the RG-improved gauge action and the clover-improved Wilson quark action on the background of $S U(3) \times U(1)$ fi elds in the quenched approximation. After chiral and continuum extrapolations, we found $\pi^{+}-\pi^{0}$ and $\rho^{+}-\rho^{0}$ mass differences are consistent with experimental values, and we can extract up, down and strange quark 

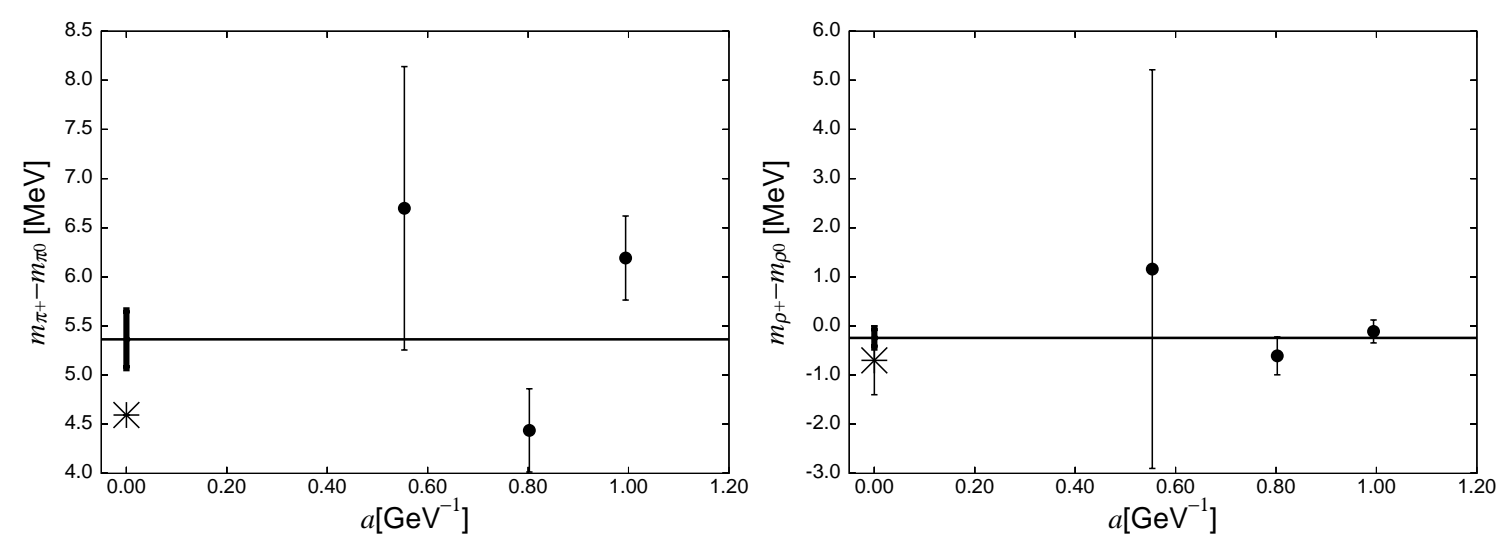

Figure 4: Lattice spacing dependence of electromagnetic mass splittings. Star represents the experimental value.

masses. We also confi rmed that mass differences obtained with $L=2.4 \mathrm{fm}$ are not shifted by fi nite size effects. An important future work is to include dynamical quark effects. Simulations using full QCD data generated by CP-PACS collaboration are ongoing.

\section{Acknowledgments}

We thanks M. Harada, M. Hayakawa, M. Tanabashi, N. Yamada and K. Yamawaki for valuable discussions. A part of our calculations have been carried out on a Hitachi SR8000 at KEK, a cluster machine at Center for Computational Sciences, University of Tsukuba, and a supercomputer (NEC SX-5) at Research Center for Nuclear Physics, Osaka University. This work is supported in part by $21^{\text {st }}$ Century COE Program, Nagoya University.

\section{References}

[1] For a recent review, see T. Izubuchi, these proceedings.

[2] C. Aubin et al., Phys. Rev. D 70031504 (2004); Phys. Rev. D 70114501 (2004).

[3] A. Duncan et al., Phys. Rev. Lett. 763894 (1996).

[4] N. Yamada et al., these proceedings.

[5] E. Dagotto et al., Nucl .Phys. B 331500 (1990).

[6] M. Harada and K. Yamawaki, Phys. Rept. 3811 (2003).

[7] S. Aoki, K. Nagai, Y. Taniguchi and A. Ukawa, Phys. Rev. D 58, 074505 (1998);

Y. Taniguchi and A. Ukawa, ibid. 58, 114503 (1998).

S. Aoki, R. Frezzotti and P. Weisz, Nucl. Phys. B 540, 501 (1999).

[8] R. Dashen, Phys. Rev. 1831245 (1969).

[9] J. Bijnens, Phys. Lett. B 306343 (1993). 